\title{
The Therapeutic Effects of Independent Eating for the Severely Physically Disabled
}

\author{
MiCHELE EISEMANN SHIMIZU, RPT, $\mathrm{PhD}^{1)}$, AKIRA OTSUKA, RPT, $\mathrm{PhD}^{1)}$, \\ SHUSAKU KANAI, RPT, MS ${ }^{1)}$, SADAAKI OKI, MD, PhD ${ }^{1)}$ \\ 1) Department of Physical Therapy, Faculty of Health Sciences, Hiroshima Prefectural College \\ of Health Sciences: 1-1 Gakuen Machi, Mihara City, Hiroshima 723-0053, Japan. \\ TEL +81-848-60-1215
}

J. Phys. Ther. Sci.
16: $73-79,2004$

\begin{abstract}
Independence in eating is one of the goals in the therapeutic intervention of people with severe disabilities of the upper extremities, who lack the voluntary movements and are completely dependent upon others to feed them. We performed a three-dimensional motion analysis using the Vicon system on a normal subject and found that the use of chopsticks and forearm supination/pronation are critical components. These were incorporated in a meal manipulator to help promote ease and accuracy of control of the device. The manipulator included a base that made positioning of the manipulator flexible; a swing arm that moves to the same point each time; a terminal device that holds the half-spoon-shaped chopsticks and produces supination/pronation movements; a table that moves the food into position for reaching; and, a control box that the user can manipulate by mouth or chin. Consequently, the user is not fed but uses his/ her remaining functions to eat independently. The manipulator was evaluated on two high level quadriplegic subjects, who provided valuable feedback. The authors concluded that independent eating using a meal manipulator could be very beneficial psychologically. Collaboration between engineers and therapists may lead to more independence for the disabled.
\end{abstract}

Key words: Independent eating, Upper extremity disability, Meal manipulator

(This article was submitted Mar. 16, 2004, and was accepted Apr. 12, 2004)

\section{INTRODUCTION}

Independent eating is something that most cultures consider an important activity in daily life. It is not only a function that is necessary to sustain life by providing the energy necessary to keep us moving and keep us alive, but is also a cultural phenomenon. "Eating activity influences many aspects of our overall medical, physical, and social well being"1). We also eat foods that are considered healthy and defined as necessary by the culture we live in. Children are trained early in their lives to eat by themselves and to use the tools provided by their cultures. When a person develops a disability that makes him/her unable to use the upper extremities for eating, the usual result is that the person becomes totally dependent on a caregiver to be fed ${ }^{2)}$. The joys of eating and being able to eat by oneself are taken away from him/her. This can lead to feelings of shame, discomfort, loss of appetite, decreased self-esteem, and panic or fear ${ }^{1,3)}$. He or she is unable to control or chose what or when he or she eats. He or she most likely is unable to relish meals, and this situation can become psychologically devastating and dehumanizing.

Independence in activities of daily living is an important part of therapeutic intervention, and, for those who are unable to feed themselves, the use of a feeding device can have good psychological value and minimize the need of assistance ${ }^{4}$. However, there has been very little in the literature relating to the use of feeding equipment to assist severely 


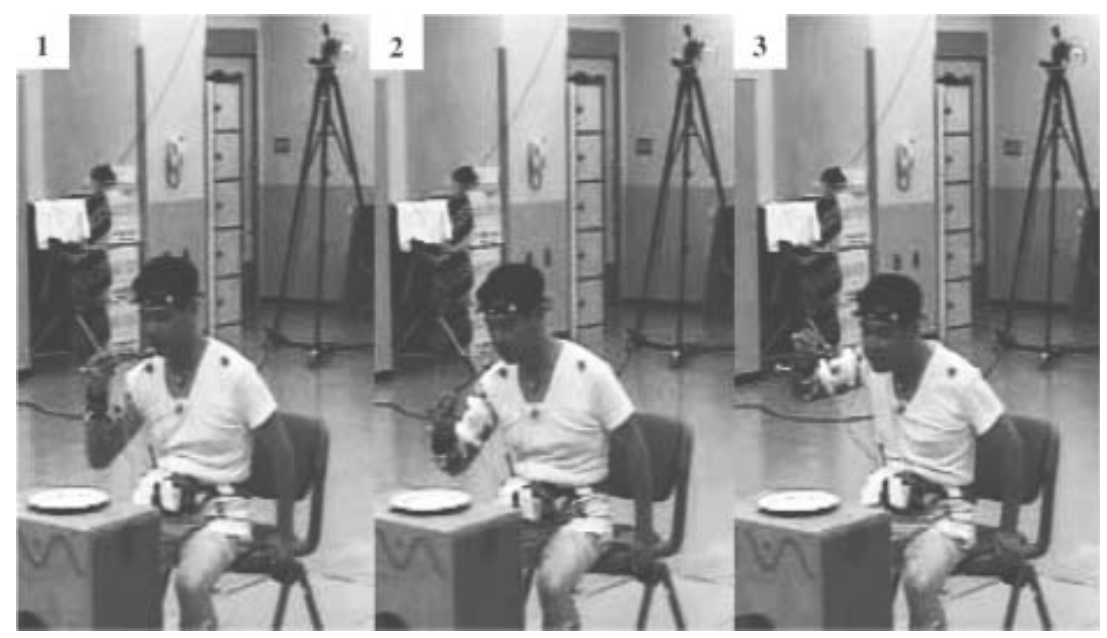

Fig. 1. Experimental conditions 1, 2, and 3. Condition 1 involved normal, free movements of the upper extremity. Condition 2 involved the elbow joint fixed at 90 degrees and free supination/pronation. Condition 3 involved fixed 90 degrees elbow flexion and fixed mid-position supination/pronation.

disabled persons. In the 1970's in the USA and in the 1980's in Japan, experiments using powered devices for feeding were begun. In 1989 and 1990, small robots and voice controls were used for meal manipulators. In 1992, "Handy 1 Feeder" (United Kingdom $)^{1)}$ made and sold meal robots. Air compressors were also used for meal robots. In the 1990's, some powered feeding devices were developed in Japan. But, it has been noted that these devices tend to be too expensive, too big, difficult to use, unreliable, sometimes unsafe, inconvenient, difficult to transport, difficult to adjust, unattractive, and time-consuming to set up $^{1,5)}$. Otsuka et al. ${ }^{6)}$ developed a meal manipulator, using actual patients as subjects to test the equipment they constructed.

By observing progressive muscular dystrophy patients for several years, it was noted that, even though the use of the upper extremities gradually deteriorates, these patients are able to feed themselves throughout their lives ${ }^{6}$. As a result of these observations, it was concluded that the use of the fingers and forearm supination/pronation and the use of chopsticks are important functions necessary for independent eating. We evaluated the meal manipulator developed by Otsuka et al. ${ }^{6}$, because it includes these functions and enabled several patients with no functional use of their upper extremities to eat independently.

The purpose of our study was threefold: to evaluate the normal upper extremity movements that are necessary for eating, to evaluate the meal manipulator used in the pilot study, and to verify our results through actual use by severely disabled, spinal cord injury persons.

\section{METHODS}

\section{Measurement of eating motions}

A meal manipulator developed by one of the coauthors in a pilot study was used ${ }^{6}$. To further develop and improve the meal manipulator, we performed measurements involving the use of Japanese chopsticks by a normal male subject. The subject was required to wear shorts and a thin Tshirt (Fig. 1).

The Vicon 512 three-dimensional motion analysis system (Vicon Motion Systems, Oxford, UK) and Body Builder software were used to analyze the data that was transcribed into stick pictures for analyzing the eating patterns of the subject (Fig. 2). This system utilized six infrared cameras located at angles around the eating position to record the movements. The data was recorded at $120 \mathrm{~Hz}$. Markers were placed in the following positions: head (4 points); trunk (C7, T10, jugular notch, xyloid process); right upper extremity (acromion, olecranon, ulnar styloid process, radial styloid process, first metacarpal); pelvis (bilateral anterior superior iliac spines, mid L5 and S1); and 


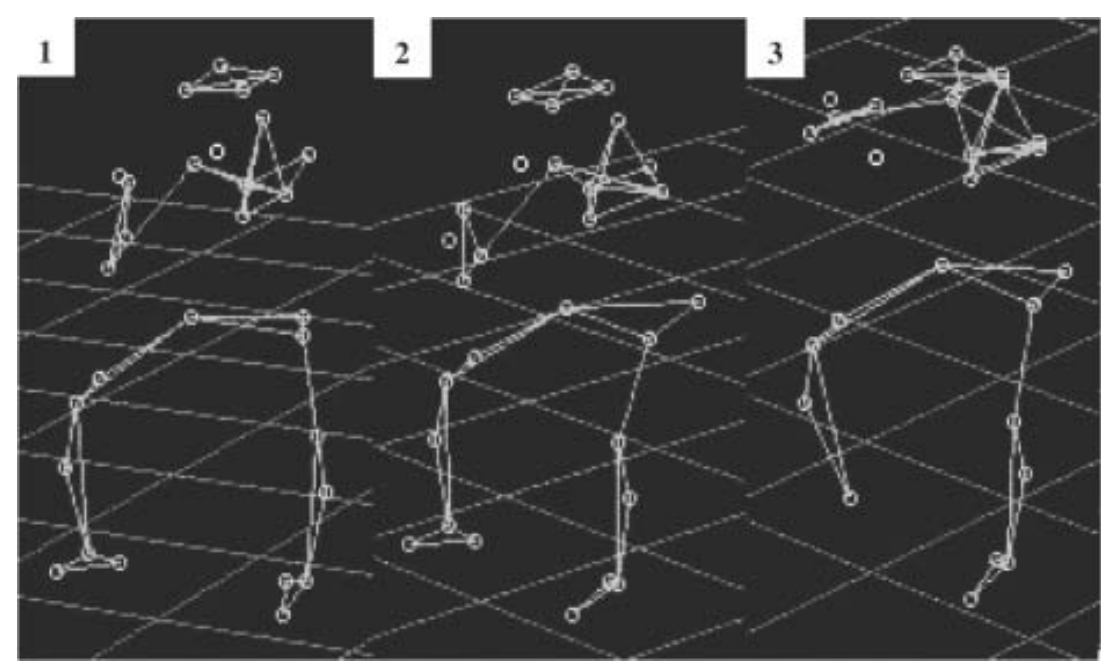

Fig. 2. Stick pictures for motion analysis. Condition 1 involved normal, free movements of the upper extremity. Condition 2 involved the elbow joint fixed at 90 degrees and free supination/pronation. Condition 3 involved fixed 90 degrees elbow flexion and fixed mid-position supination/pronation.

bilateral lower extemities (mid lateral femur, mid lateral knee joint, mid lateral lower leg, lateral malleolus, heel, first metatarsal). In addition, the eating trial was also videotaped by a standard digital video cameral (DCR-PC100, SONY) at $30 \mathrm{~Hz}$ located towards the center of the eating position to assist with the analysis of the data. The acromion and olecranon were used as key points to observe the movements for analysis. A brace (Nakamura Brace, Japan) was used to limit elbow motion.

A three-dimensional movement analysis was done under three conditions for the elbow joint and forearm supination/pronation. Condition one (1) involved normal, free movements of the upper extremity. Condition two (2) involved the elbow joint fixed at 90 degrees and free supination/ pronation. Condition three (3) involved fixed 90 degrees elbow flexion and fixed mid-position supination/pronation (Fig. 1). Elbow flexion was fixed at 90 degrees, because it is the angle at which a person can easily bring the hand to the mouth.

\section{The meal manipulator}

Our meal manipulator (Fig. 3), still in the experimental stage, was tried on two spinal cord injured quadriplegic subjects. The construction is described below.

i) Meal manipulator base

The function of the base (Fig. 4) is to move the

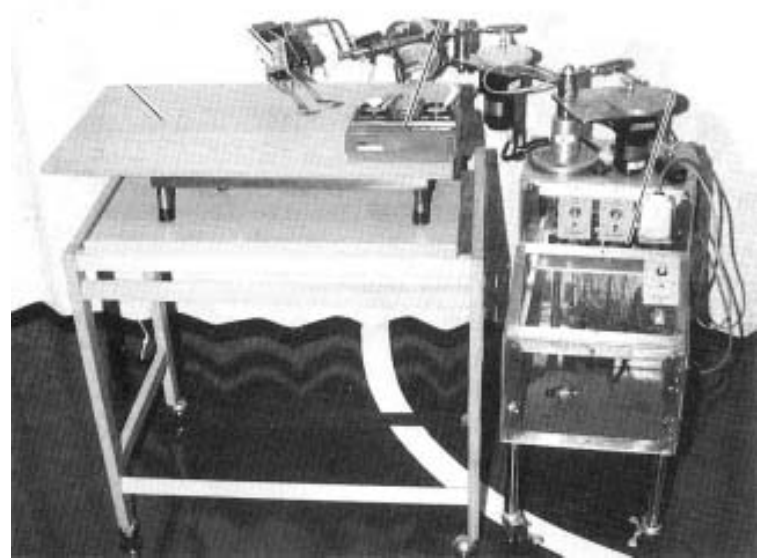

Fig. 3. Meal manipulator.

terminal device and adjust it to the best position for feeding. Motor one (1M) regulates the tilt of the device. Motor two (2M) regulates the distance. Motor four (4M) controls vertical movements. The caregiver makes the adjustments to provide the subject with the best position for feeding. Motor three (3M) moves the swing arm in one set pattern. For further safety, the length of the total link system is limited by a "limit switch" attached to the joints for Motor two and Motor three of the swing arm. The degree of limitation is adjusted to each person, and this prevents the patient from being hit by the 


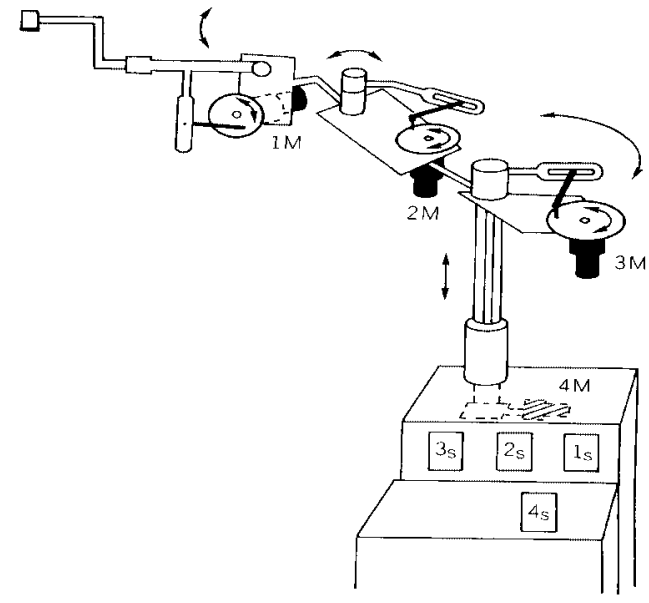

Fig. 4. Meal manipulator mechanism.

chopsticks. In addition, the subject can switch the manipulator on or off with his chin or mouth. When one turns the switch off, the arm stops where the subject wishes it to stop. Another switch is then used to produce supination and pronation to pick up the food to be eaten. The speed of the movement can be adjusted by the caregiver.

To determine the amount of force used by the device in "supination" and "pronation" to get the food up to the user's mouth, a sensor able to measure a maximum of $5.0 \mathrm{~kg}$ was attached to a Digital-Hand Dynamometer (Sakai Medical Company, Ltd., Japan). The manipulator was found to be able to produce a maximum supination force of $4.0 \mathrm{~kg}$, and it was concluded that the device was fully capable of lifting food from the table up to the user.

\section{ii) Terminal device}

Motor A (Fig. 5) controls supination/pronation of the chopsticks. Motor B holds and releases the chopsticks. We chose chopsticks shaped like halfspoons to enable the subject to scoop as well as hold food. As the chopsticks are pulled towards the motor (b2), they close and pick up food (b3). When the chopsticks are pushed out, they open. The pinch strength of the chopsticks was tested by a sensor attached to the Digital-Hand Dynamometer and was found to be capable of holding up to a maximum of $1.2 \mathrm{~kg}$ (maximum amount measurable by the sensor is $2 \mathrm{~kg}$ ). We found that in healthy persons using chopsticks with their hand, a maximum pinch strength of $0.6 \sim 0.8 \mathrm{~kg}$ is necessary to use them effectively. Therefore, we concluded that the
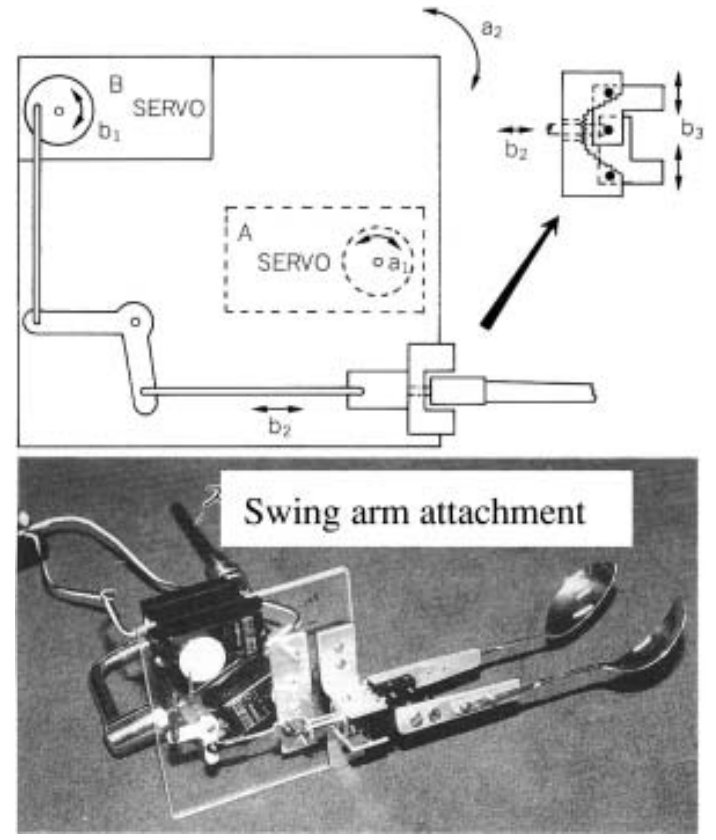

Fig. 5. Terminal device.

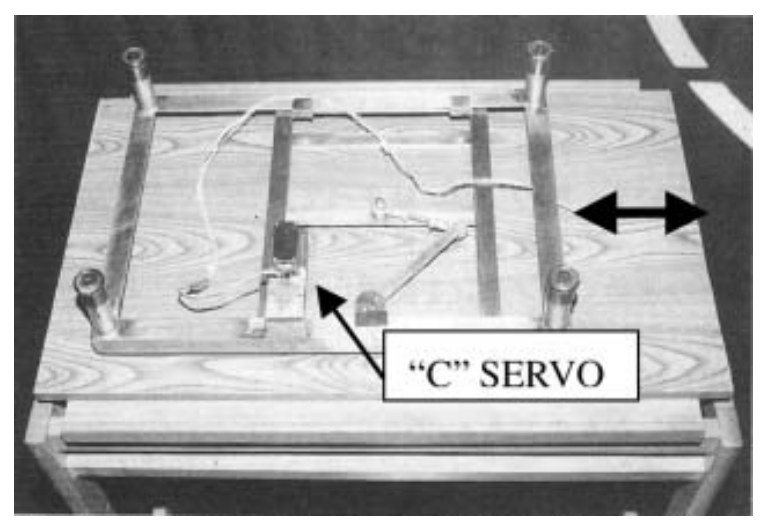

Fig. 6. Dinner table set-up.

chopsticks attached to the meal manipulator are effective for use in a feeding device.

iii) Dinner table

As seen in Fig. 6, the table is moved sideways by a motor. The motor is based on a link system to enable the table to slide. The terminal device only swings and picks up food, so the table was devised to slide and move the food into position for food selection.

iv) Control box

A wired four-channel box was used to make the 

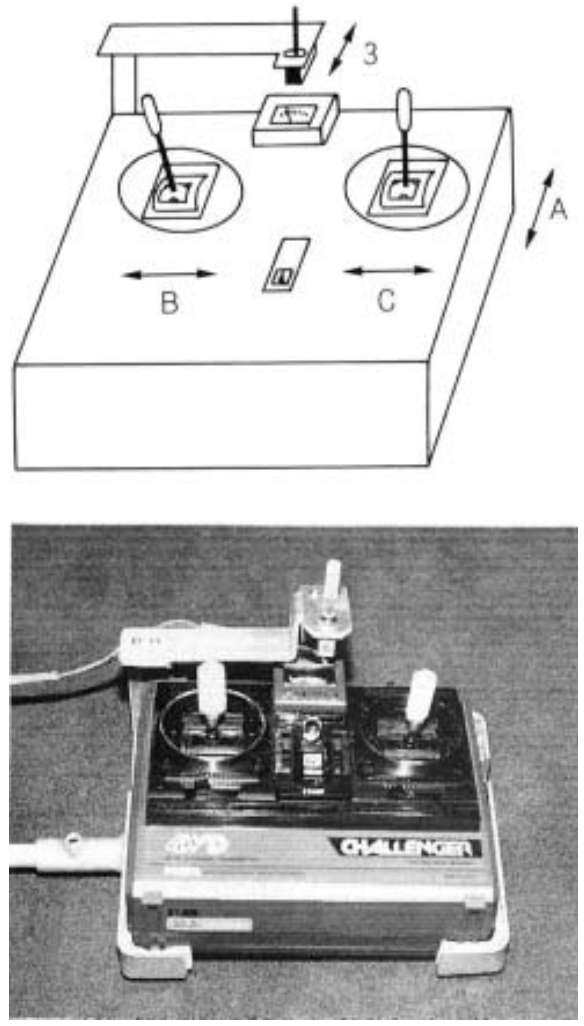

Fig. 7. Control box.

control box (Fig. 7). "A" controls supination/ pronation, and "B" controls the movement of the chopsticks. "C" controls the sliding of the table. (The fourth channel will be used when further improvements to the manipulator are done.)

Another switch (3) is the on/off control for the swing arm. All the switches are light and easy to move, so cervical movements are kept to a minimum.

\section{Subjects}

Two high level spinal cord injured persons volunteered to be convenience samples for this study. The subjects were their own controls as they used the manipulator and compared its use to their daily situation of being fed. The authors screened the subjects for their suitability for evaluating the device. The selection factors included a severe disability that precluded independence in eating, adequate vision and head control to enable them to operate the equipment, and a cognitive ability to use the manipulator and give their opinions about $\mathrm{it}^{1,5}$ ). The subjects were taught how to use the device, after which they were presented with open-ended

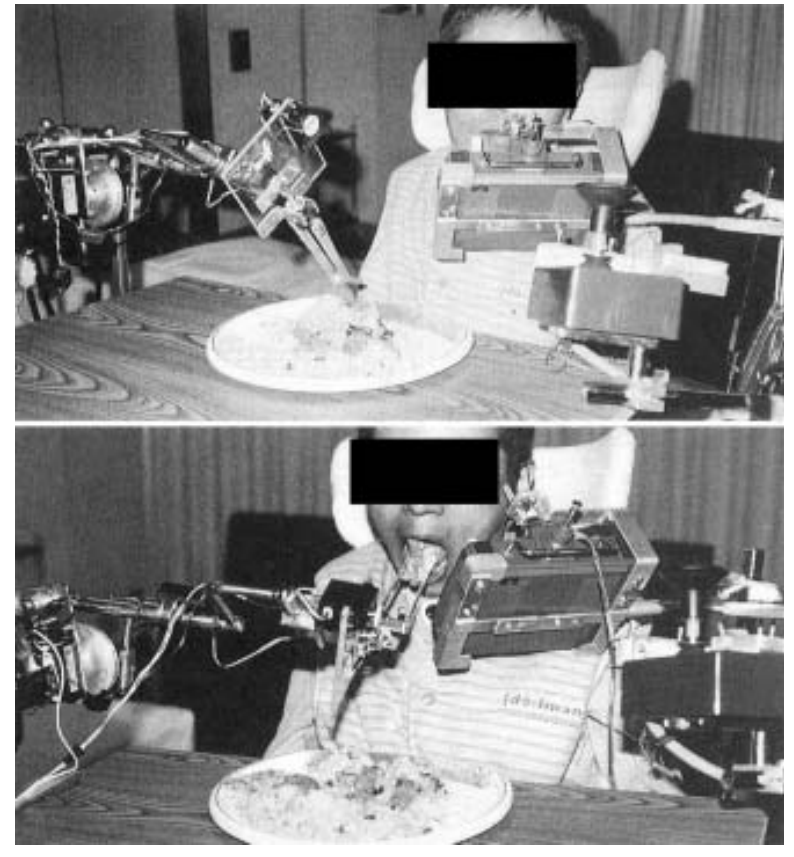

Fig. 8. Case one while using meal manipulator.

oral questions on the ease and practicality of its use. The two subjects included the following attributes.

i) Case one (Fig. 8)

This male subject had a C3 spinal cord injury three years prior to this study, which left him with no voluntary use of his trunk, arms and legs. Before the accident while "wrestling", he was a road construction worker. He was 35 years of age at the time of this experiment. During the experiment he was an in-patient in a university hospital. His only diagnosis was a spinal cord injury, and he had a tracheotomy and used a respirator for respiratory distress. He could not sit by himself, so he used a special bucket seat to keep him upright in his electric wheelchair. However, he could only sit for about two hours at a time with rests in between. He had good head and neck control but only a slight shoulder shrug. He used his chin to control the movements of his wheelchair. Total care for all his activities of daily living was necessary, including being fed by caregivers.

\section{ii) Case two (Fig. 9)}

This 30 year-old spinal cord injured male had a right $\mathrm{C} 5$, left $\mathrm{C} 4$ injury as a result of a car accident over 10 years earlier. Before the accident, he was a 

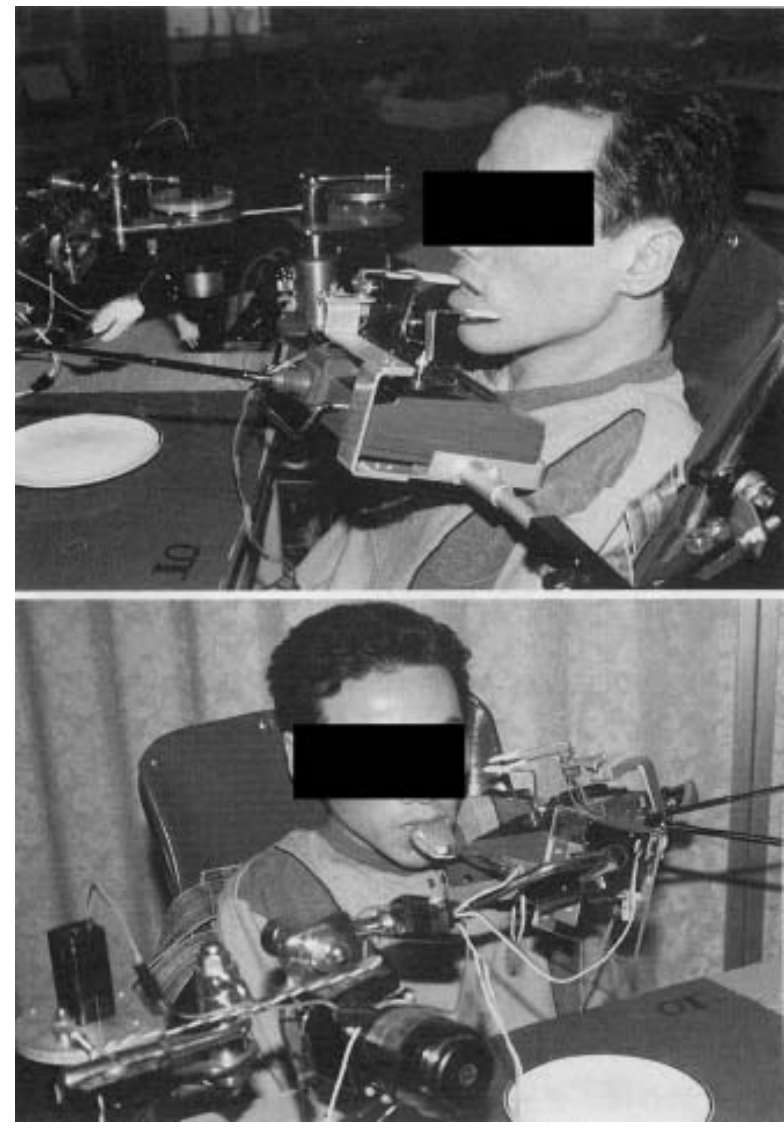

Fig. 9. Case two while using meal manipulator.

car salesperson. At the time of this study, he was a patient in a rehabilitation center, and his only diagnosis was spinal cord injury. No respiratory problems were noted. His blood pressure was low, so he needed to sit slightly reclined (about 45 degrees). He could not sit up straight or for more than an hour without needing a rest. He had good head and neck control and a fair shoulder shrug. Total support for activities of daily living was essential. He had tried many feeding devices before using ours, but to no avail.

\section{RESULTS}

\section{Motion analysis of single subject during eating}

i) Vertical movements of the acromion

As seen in Fig. 10, under Condition one (1), the movement of the acromion is small and tends to depress somewhat during eating. Under Condition two (2), the acromion depresses even more, but the amount of movement is still minimal. These results

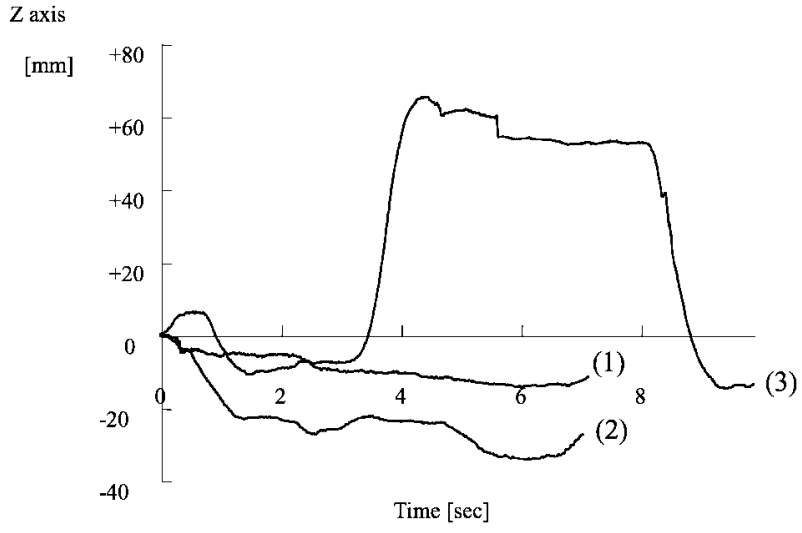

Fig. 10. Acromion vertical movement graph analysis.

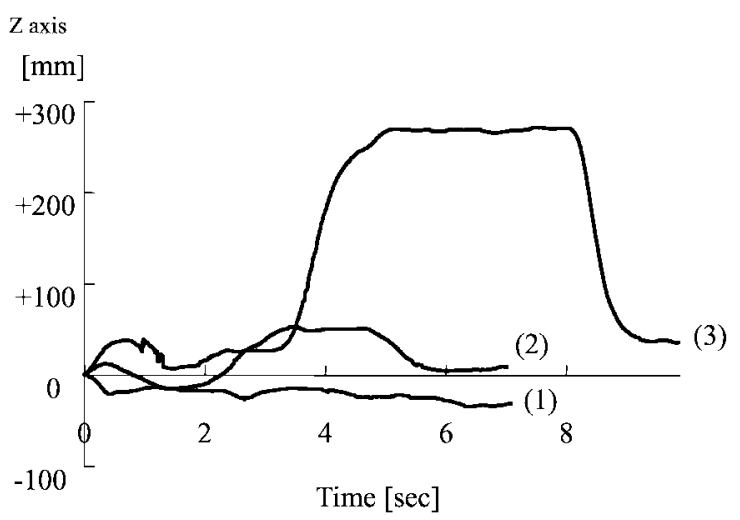

Fig. 11. Olecranon vertical movement graph analysis.

tend to indicate that supination and pronation is used to keep shoulder movements to a minimum. As can be seen in the figure, under Condition three (3), the trick motion of the shoulder is extreme, most likely due to the limitation of supination and pronation.

ii) Vertical movements of the olecranon

Under Condition one, as seen in Fig. 11 (1), the movement of the olecranon is minimal and slightly depressed, as is the acromion. Under Condition two (2), the movement is slightly larger, and compensatory movements appear necessary to accommodate the fixation of the elbow. However, under Condition three (3), again there is an extreme vertical movement of the elbow joint to compensate for the lack of supination/pronation. The curves for Conditions two and three are similar, but the presence or lack of supination/pronation makes a difference in the amount of compensation. 


\section{CASE REPORTS}

Case one stated that he desired to be able to chose his foods and eat by himself, even if it meant spilling some of his food (noodles and soup). He was very pleased to be able to use the meal manipulator successfully.

Case two used our meal manipulator, but all the switches had to be extended perpendicularly to enable him to reach them from his reclined position. He was extremely happy about being successful in eating even Japanese sushi.

When fed by a caregiver, usually the person feeding decides on when and what to feed the person being fed. Consequently, both subjects agreed that being able to choose what they want to eat and when they want to eat it, without having to depend on someone to feed them and always be anxious about the caregiver's feelings, was the most beneficial aspect of using this device. They both showed enthusiasm about the personal control over the speed and order of eating. In addition, the caregiver does not have to rearrange the food to facilitate eating, because the table moves the food into position as the eater manipulates it with the control switch. Both subjects agreed that the meal manipulator was easy to use, even after just a few practice sessions. However, because the "chopsticks" are actually half-spoons that are joined together through a control switch, leakage can occur through the matched edges. This results in the spilling of soup and noodles, which both subjects pointed out as disadvantages of this device.

\section{DISCUSSION AND CONCLUSION}

Several different methods for assisting the disabled in feeding themselves have been tried in the past, including orthotics, adaptive equipment, braces, and powered feeding devices ${ }^{1)}$. However, the usefulness of any device depends on the type of disability, muscle strength, joint range of motion, coordination, remaining functions, energy cost, subject comfort and convenience ${ }^{1)}$. The long-term side effects of any piece of equipment are inevitable and vary with the subject and the equipment itself. The ultimate goal of adaptive equipment is that it reduces energy cost and increases independence and self-esteem.
In this report, we have introduced a meal manipulator, which was originally developed for a pilot study done by one of the co-authors ${ }^{6)}$ and is still in the experimental stage. A three-dimensional movement analysis with a Vicon system was done on a normal subject to verify the reason why muscular dystrophy patients continue to be able to feed themselves and to establish the viability of the meal manipulator used in the pilot study. As a result of these experiments, we concluded that including a supination/pronation function and "chopsticks" for self-feeding are essential. Therefore, we believe that the inclusion of supination/pronation in our meal manipulator is extremely important. The pilot study feeder included these functions, so two case studies were used to test it clinically. The highlevel spinal cord injured subjects were able to eat meals independently and, thus, gained psychological benefits. Both subjects stated that they were very satisfied with the manipulator and that they would like to continue using it. We hope to try our meal manipulator on more subjects, different disability populations, for longer periods of time, and to improve it as we go along.

In conclusion, we would like to continue our work in developing various equipment that may be used for the rehabilitation of severely disabled persons. For better results, we believe that collaboration between engineering experts and medical team experts, including physical therapists, is essential.

\section{REFERENCES}

1) Hermann RP, Phalangas AC, Mahoney RM, et al.: Powered feeding devices: an evaluation of three models. Arch Phys Med Rehabil, 1999, 80: 12371242.

2) Wyckoff E, Mitani M: The spoon plate: a self-feeding device. Am J Occup Ther, 1982, 36: 333-335.

3) Perry L, McLaren S: Eating difficulties after stroke. J Adv Nurs, 2003, 43: 360-369.

4) Hall W, Hammock M: Feeding and toileting devices for a child with arthrogryposis. Am J Occup Ther, 1979, 33: 644-647.

5) Seamone W, Schmeisser G: Early clinical evaluation of a robot arm/worktable system for spinal-cordinjured persons. J Rehabil Res Dev, 1985, 22: 38-57.

6) Otsuka A, Takahashi M, Amano $H$, et al.: Development of an eating function support systemespecially for severely disabled persons. Rigaku Ryoho Journal, 1991, 25: 53-57 (in Japanese). 